\title{
Instruments and Players:
}

\section{Some thoughts on digital lutherie}

\author{
Sergi Jordà \\ Music Technology Group, Audiovisual Institute, Pompeu Fabra University \\ Ocata 1, 08003 Barcelona, Spain \\ Tel.:+ 34935422104 \\ E-mail: sergi.jorda@iua.upf.es
}

\begin{abstract}
Music instruments are used to play and to produce music, transforming the actions of one or more performers into sound. This article explores some instrument design issues in three distinct parts. The first section attempts to define what music instruments are, how traditional instruments function and what they can do, and what future instruments could be, trying to figure out how we could better exploit their unlimited potential. The second section proposes a quick review of the different know-how, the technical and the conceptual frameworks and areas in which new instrument designers and researchers are currently working on. It is not in that sense, a survey of new instruments and controllers but more a survey of thoughts and knowledge about them. The third and last section studies the dynamic relation that builds between the player and the instrument, introducing concepts such as efficiency, apprenticeship and learning curve. It explores some music instruments generic properties such as the diversity, the variability or the reproducibility of their musical output, the linearity or non-linearity of their behavior, and tries to figure out how these aspects can bias the relation between the instrument and its player, and how they may relate to more commonly studied and (ab)used concepts such as expressivity or virtuosity. The aim of this paper is the foundation of a theoretical framework in which the possibilities and the diversity of music instruments as well as the possibilities and the expressive freedom of human music performers could all be evaluated; a framework that could help to figure out what the essentials needs for different types of musicians -from the absolute novice to the professional or the virtuoso- may be.
\end{abstract}




\section{Introduction}

Music instruments are used to play and to produce music, transforming the actions of one or more performers into sound. New digital instruments design is a quite broad subject, which includes highly technological areas (e.g. electronics and sensor technology, sound synthesis and processing techniques, computer programming...), human related disciplines (associated with psychology, physiology, ergonomics and many human-computer interaction components), plus all the possible connections between them (e.g. mapping techniques...), and the most essential of all, music in all of its possible slopes. Because new instruments' designers may be partially responsible for the music to come, when envisaging new instruments, designers do not have to their study to the instruments' sonic capabilities (which can be absolutely any), not even to their algorithmic power; they must be also especially careful about the instruments' conceptual capabilities, to the ways instruments impose or suggest to their players new ways of thinking, new ways of establishing relations, new ways of interacting, new ways of organizing time and textures; new ways, in short, of playing new musics.

Often, a new instrument is derived directly from an existing instrument... We claim that this transfer will often result in similar music from the two instruments, however. Conversely, one method for making truly new music might be to create an instrument that allows performers to experiment with new playing techniques (Rubine \& McAvinney, 1990).

Given the relevance of musical instruments in music creation it is therefore no surprise that researchers from the most diverse computer music disciplines claim to be working with or on them. I came to this platitude last summer at SMAC $2003^{1}$, where I was invited to give a talk on innovative musical instrument control. In my main research area, which roughly verses around new interfaces for musical expression (NIME), we do often employ the term "musical instruments" when we are frequently referring only to input devices or controllers. In Stockholm, I did also realize that sound synthesis researchers were invoking this term as well, but applying it to sound generation methods and algorithms. It should be made obvious that both sectors are equally wrong; half wrong to be more precise, or only half right if we want to be benevolent. NIME people focus on the input-playing side while acousticians and synthe sists concentrate on the output-sounding one. Sound synthesis (and interactive real-time synthesis and processing, as a particular case with specific technological needs and restrictions) is a well settled technical discipline, entering its fifth decade, with important congresses (e.g. DAFX ${ }^{2}$ ) and thousands of articles and books being published every year. In recent literature a great deal of interest is also being deployed on new alternate controllers and the same can be said of algorithmic composition and interactive music systems. But the fact is that very few attempts are being made at studying the design of new musical instruments -tools for playing and making music- as a conceptual whole; none of the

\footnotetext{
1 SMAC03, Stockholm Music Acoustics Conference 2003, The Music Acoustics Group at the Department of Speech, Music and Hearing, KTH, August 6 - 9, 2003, http://www.speech.kth.se/smac03/

${ }^{2}$ DAFX, Digital Audio Effects Conference, http://www.dafx.de/
} 
aforementioned disciplines is giving by its own too many clues on what instruments we could invent, why, how interesting they could be or how we could try to make them better.

Musical interface construction proceeds as more art than science, and possibly this is the only way it can be done (Cook, 2001).

Let me close this introduction with a warning. Digital lutherie is in many senses, very similar to music creation. It involves a great deal of possible and different know-how, the use of many technical and technological issues but, like in music, there are no inviolable laws. That is to say that digital lutherie should not be considered as a science, no more than music, but as a sort of craftsmanship, that may sometimes produce -in very exceptional cases- a work of art; no less than music.

\section{Traditional and Digital Music Instruments}

\subsection{The instrument chain: Controllers and Generators}

An acoustic instrument consists of an excitation source that can oscillate in different ways under the control of the performer(s), and a resonating system that couples the vibrations of the oscillator to the surrounding atmosphere, affecting, at its turn, the precise patterns of vibration. Parts of the performer body transmit to the instrument the energy for the excitation, interacting through the instrument's control interface. However, in most acoustic instruments (apart from organs and several other key instruments), this separation between the control interface and the sound-generating subsystems is fuzzy and unclear (Hunt, Wanderley \& Kirk, 2000; Wanderle y, 2001).

Digital musical instruments on their side can always be easily divided into a gesture controller or input device that takes the control information from the performer(s) and a sound generator that plays the role of the excitation source. The musical controller (or input device), is a piece of equipment that the player uses to control how the sounds stored or created in real time by the computer will be released. This separation between gesture controllers and sound generators was standardized by MIDI two decades ago, and it sprouted the creation and development of many new alternative controllers with which to explore new creative possibilities (e.g. Waisvis z, 1985; Chadabe, 1997; Wanderley \& Battier, 2000). It also incremented musical flexibility; by allowing any controller or input device to control any sound generator, MIDI became the "crossbar switch" between gesture and timbre (Rubine \& McAvinney, 1990).

\section{Splitting the chain: Drawbacks}

But this separation has also some significant negative counterparts, the most obvious being the reduction of the 'feel' associated with producing a certain kind of sound (Roads, 1996). Another frequent criticism deals with the own limitations of the protocol that connects these two components of the instrument chain. Created twenty years ago but still ubiquitous, MIDI is undoubtedly restrictive and restricted for today's benchmarks. It offers low bandwidth, low resolution, an inaccurate timing, half-duplex 
communications, an inflexible mapping scheme, etc., many of these limitations being already pointed out soon after its birth (Moore, 1988).

There is however a more essential drawback concerning the conceptual and practical separation of potentially new digital instruments into two separated components: it becomes hard -if not impossible- to design highly sophisticated control interfaces without a profound prior knowledge of how the sound or music generators will work. Generic or nonspecific music controllers tend to be either too simple, mimetic (imitating traditional instruments) or too technology biased (i.e. they are constructed because they can be constructed). They can be inventive or adventurous but how can they, in these cases, guaranty coherence, if they cannot anticipate what their controllers are going to control?

And inadequate controllers will fatally lead to unsuccessful instruments.

One has to think of overall systems to get a musically useful thing-you can't really develop a sensor without relating it to the programs that you're going to use it with (Max Mathews, excerpt from Chadabe 1997, p. 230).

From the "generator" side of the glass, these design limitations may not seem so obvious at first glance. Researchers working on physical model synthesis might have reasons to believe that the corresponding optimum controllers will probably be digital simulations of their real counterpart (i.e. a MIDI guitar or a MIDI single-reed wind controller, for example) (e.g. Serafin et al., 1999).

Are we suggesting therefore that when envisaging new digital instruments, the conception of the generator should always come first, subsequently followed by a controller design made to measure? Not at all! For several reasons. First, although this approach surely makes sense for many sound synthesis methods based on physical models, sound synthesis is not limited to physical modeling. As Perry Cook suggests, there are many cases in which a discovery within a synthesis algorithm development suggests a new control metaphor ${ }^{3}$, or where a given control component suggests new aspects of a synthesis algorithm (Smyth \& Smith, 2001; Cook, 2003). But even if this synthesizer $\rightarrow$ controller design approach was still applicable to any kind of synthesis (in many cases it could still be perfectly justifiable), a more crucial point is that new musical instruments output is not limited to sound synthesis.

New instruments are not forced to remain at the sound and note level; with the added "intelligence" that computers can bring them, new digital instruments can also embrace algorithmic composition, they can deal with tempo, with multiple and otherwise conventionally unplayable concurrent musical lines, with form, they can respond to a performers in complex, not always entirely predictable ways (Chadabe, 1984). How could a best design strategy be, in these untraditional (and highly desirable) cases?

As we already anticipated, an ambitious goal for any new instrument is the potentiality to create a new music. In that sense, baroque music cannot be imagined without the advances of $\mathrm{XVI}^{\text {th }}$ and XVII ${ }^{\text {th }}$ century luthiers, rock could not exist without the electric

\footnotetext{
${ }^{3}$ One of the examples illustrated by Cook is indeed based on physical model synthesis. The fact that it was a model for voice synthesis, made hard to mimic the "original" controller. As a result, the SqueezeVox, a concertina-inspired controller for voice-synthesizer was born (Cook \& Leider, 2000).
} 
guitar, or jazz and hip-hop, without the respective redefinition of the saxophone and the turntable (Settel \& Lippe, 2003). For that reason, if we believe that new musical instruments can be partially responsible for shaping some of the future music, if we seek to create new sophisticated musical instruments aiming at creating new music-making paradigms, a parallel design between controllers and generators is needed, with both design processes treated as a whole.

To better understand both aspects, we will try to answer two basic questions that can be applied to all kinds of instruments, included all traditional ones: what can they do (output), and how can they be played or how these sonic possibilities are attained (input).

\subsection{Acoustic Instruments: a Quick and Dirty Overview}

\section{Outputs}

While traditional instruments may differ enormously from one family to another, their simplified possibilities (what they can do) can be summarized in a short list.

- Start playing a tone with a given pitch, a given dynamic and a given timbre. Some instruments have a continuous choice of pitches, while in others this choice is discrete or even inexistent. The same applies to dynamics and to timbre (i.e. continuous, discrete or unique dynamics and/or timbre).

- In polyphonic instruments, this process can be started concurrently several times, while in monophonic instruments the current tone has to end before a new one starts.

- In some instruments the tone stops automatically after a short time. Other instruments are capable of sustaining the tone at the player's will ${ }^{4}$.

\section{Pitch variation}

- When the pitch space is continuous, changes can be applied to it, basically in three ways:

- Glissando (continuous variation from one pitch to another). By using this mechanism, monophonic instrument can go from one tone to another without stopping in between (portamento).

- Vibrato (small and fast oscillating shifts).

- Trills (fast oscillations between two or more tones).

- In many instruments, pitch can be considered continuous within a short region or range (e.g. guitar, trumpet). In that case, vibrato and trills are possible, but not glissandi.

- When pitch is discrete, trills are the only pitch changes allowed.

\section{Dynamics variation}

- When the dynamic space is continuous, permanent changes can be applied to it in two basic ways:

- Tremolo (small and fast oscillating shifts).

○ Continuous variation of dynamic (no specific word for it).

\section{Timbre variation}

- Very few instruments allow for a continuous control of timbre (e.g. voice, didgeridoo).

\footnotetext{
${ }^{4}$ In the XVIth century, Praetorius had already classified instruments according to three quantitative characters: (1) how long the instrument can hold a tone and whether its tuning is fixed or not; (2) whether it can be made to produce all the tones and voices of a piece or only some or only one voice at a time; (3) whether it can play notes beyond its natural range or not (Praetorius, 1980).
} 
- Many instruments offer however different discrete timbres, either because they combine separated parts (e.g. percussion kits), or because they can be played using different techniques (e.g. violin) or with different accessories (e.g. sticks or mallets).

- Several instruments offer also different timbre regions resulting from the instrument morphology, or permit reduced timbre and spectral effects (such as the use of mutes in brass instruments).

- In the remaining ones, timbre is strongly dependent on the other two parameters, pitch and dynamics.

Table 1. A quick and dirty acoustic ins truments possibilities overview

Each instrument or family of instruments favors some of these possibilities over others ${ }^{5}$. It should also be noticed that polyphonic instruments have always had limited continuous control possibilities (Pressing, 1990; Rubine \& McAvinney, 1990; Vertegaal \& Eaglestone, 1996; Levitin et al., 2002) ${ }^{6}$ and the reasons for these restrictions have to be found in the physical and cognitive human limitations and are not inherent to the instruments (Fitts, 1954; Miller, 1956; Fitts \& Posner, 1967; Cook, 2001).

\section{Inputs}

Concerning the second question we have posed (how can they be played), acoustic instruments are not played in that many different ways. If we do not enter into nuances, instruments receive energy in four possible ways, by being blown, struck, plucked or rubbed. These ways are conditioned by the instrument construction and are determined by the instrument output capabilities ${ }^{7}$. With few exceptions (as in the already mentioned case of the organs and several keyboard instruments), acoustic instruments impose their own playability rules, which allow listeners to infer the type and form of the gesture from the sound being generated. There may still be room for some control design improvements but scarcely for capricious decisions or radical changes, which is surely not the case of digital instruments.

\subsection{The Digital Luthier's Framework: Everything is Possible}

As previously exposed, by eliminating its physical dependencies, the split in the controller-generator chain eradicates also all previous restrictions on the design of digital instruments. Any input can be now linked to any output, and each of both components can offer a virtually infinite number of possibilities of which we will only briefly suggest a few.

\footnotetext{
5 Notice that while this simplified list may be applied to any existent instrument of any culture, its exposition reflects a considerable Westerner ethnocentrism: its favors pitch (over the remaining parameters), discrete choices, and discontinuous events with a beginning and an end instead of an open and non hierarchical evolution of parameters as allowed by many instruments.

${ }^{6}$ The electric guitar could be considered an exception to this rule.

${ }^{7}$ Although the inverse would sound more logical (i.e. "the output will depend on the way an instrument is played") what we suggesting here is that, for example, if an instrument can sustain sound, it is because it can be either blown or rubbed.
} 


\section{Inputs}

On the input side, the wider and ever increasing availability of sensing technologies enables virtually any kind of physical gesture or external parameter to be tracked and digitized into a computer. What is more, the wide accessibility of devices such a video cameras, analog to MIDI interfaces (ADB I/O, ICube, Sensorlab, SAMI, AtoMIC Pro, etc.) (Wanderley, 2001), easily programmable micro-controllers such as the Basic Stamp or even analytic interfaces which hear musically, has definitely opened these plethora of input possibilities to anyone willing to explore the territories.

\section{Sonic Outputs}

The output side may cover without any restriction all the aspects included in the previous acoustic instruments possibilities list. Any parameter variation can be made now as desired, continuously, discreetly, or in a combination of both. Small periodic changes can still be applied to them, now with an unlimited and precise control over all of the oscillation parameters (frequency, amplitude and shape). More significantly, timbre does not have to be left out anymore; any (un)imaginable timbre can be theoretically rendered; it can change abruptly or evolve continuously and even smooth transitions between any set of distinct timbres can be achieved (morphs).

\section{Everything is too much}

It should be reminded again that the possibility of an instrument "to do everything" does not imply that a human player is capable of controlling it all. Digital instruments having memory and brain, these can be used to reduce some of the human control overflow; added possibilities are again endless, as we will show.

\subsection{Intelligent Instruments}

\section{Scored Instruments}

Traditional instruments can be played with a score (i.e. the actions taken on them can be notated), but except some rare cases (like the pianola) they do not embed these scores. But new digital instruments do not have to be scoreless; time lines of different kinds can be embedded on them, which may allow to control from subtle parameters nuances either fixed in time (scored) or dependent of other events- to a whole piece. When back in 1969, Max Mathews was designing the Conduct program for the GROOVE, possibly one of the first computer-based musical systems that can be considered as an instrument (Mathews \& Moore, 1969; Mathews, 1991), he choose to store the sequence of pitches to be played, after he realized that in most of the compositions, pitch was the parameter more rigidly fixed by the composer. Consequently, he GROOVE+Conduct system allowed a person to play controlling certain musical effects like amplitude, tempo, and balance over the course of an entire piece of music, but not the notes.

It ... seemed to me that there were a number of aspects of music where the performer didn't have any freedom of choice. The sequence of notes to be played, for example, was an area in which the computer could legitimately help a performer in making it easier to do things correctly (Roads, 1989, Interview with Max Mathews). 
And if an instrument is capable of knowing which note comes next, some more knowledge may enable it to take decisions on the fly. Instead of using predetermined pitch sequences, each note to be played could for instance result from the combined decisions of the player and the instrument altogether. In a be-bop improvisation context for example, the player could select pitches from a set that would contain only the permitted pitches within the current chord or mode (Nishimoto et al., 2003). This brings us to intelligent instruments, a term that was first coined by Laurie Spiegel and Max Mathews in about 1973 (Chadabe, 1997)

\section{Intelligent Instruments and Interactive Music Systems}

Intelligent Musical Instruments are instruments that first sensed its performer's intent by the type and context of the performer's actions and then extended the performer's controls to execute the music automatically. Intelligent Instrument let people play the music on a compositional level (Gagne, 1993, Interview with Laurie Spiegel).

The intelligent musical instrument can also respond to a performer in a complex, not entirely predictable way, thus forcing the performer to react on its turn, and opening the door to what Joel Chadabe defines as Interactive Composing, a term he invented in 1981 to describe a performance process wherein a performer shares control of the music by interacting with a computer music system. It is a stage that involves simultaneously composition and performance (Chadabe, 1984, 1997).

It is a characteristic of Interactive composing that a performer, in deciding each successive performance action, reacts to information automatically generated by the system (Chadabe, 1984).

Being able to play at a macrostructural level, intelligent instruments break the one gesture to one acoustic event paradigm present in all traditional instruments.

It should be clear by now that all the relief that "instrumental intelligence" can bring to enlighten human surcharge at the sound control level, can be easily swept away by the new-fangled musical possibilities that new instruments can suggest (e.g. Roads, 1986; Polansky, Rosenboom \& Burk, 1987).

I would like to terminate this section with two additional comments on intelligent instruments.

\section{Microstructural-macrostructural Musical Instrument Polarization}

When working at a microstructural level, the process translates into texture or other timbrical phenomenon; in the macrostructural one it translates into musical form. Since the second half of the $\mathrm{XX}^{\text {th }}$ century, many composers such as Iannis Xenakis (Xenakis, 1992), Giacinto Scelsi, James Tenney or Curtis Roads (Roads, 2001a), to name only a few, have been concerned with the integration of these two musical poles at the compositional level. Computers seem specially suited for bridging this gap, and some of the aforementioned composers, like Xenakis and Roads have even designed or 
conceived software tools, such as the UPIC (Marino, Raczinski \& Serra, 1990) or the PulsarGenerator (Roads, 2001b) that allow to capture both microstructural detail and macrostructural evolution But the fact is that common practice has not always encouraged the merger, as the prevalent use of the two complementary computer music omnipresent concepts score and orchestra, common to most Music N languages, can for example confirm.

This prevailing separation, apart from being anachronistic, is a burden for the blooming of new instruments capable of both sound and form control at a similar conceptual level and by means of the same gestures. An approach, which in my opinion, could open one path to truly new musics (Jordà, 2001, 2002a).

\section{Some warnings on instruments' intelligence}

There is the desire on the part of some computer music researchers to create new instruments that should "know" what to do and require very little skill to play. Though this might allow beginners and amateurs to enter into music making quickly and enjoyably, this trend might ultimately limit a person's understanding of music. The question is whether these instruments will stimulate due to their immediate accessibility, or suffocate due to a kind of atrophy of learned musicality (Schloss \& Jaffe, 1993).

Smart instruments are often not smart. For normal humans, being able to pick up an instrument with a simple obvious interaction and "play it" only makes logical sense. That the instrument is "learning from their play and modifying its behavior" often does not make any sense at all, and can be frustrating, paralyzing, or offensive (Cook, 2001).

Intelligence is a tricky thing. Use it intelligently.

\subsection{And yet...}

New instruments possibilities are indeed endless. Anything can be done and many experiments are being carried out. Yet, current situation and results can hardly be considered awesome.

- The list of new instrument virtuosi and/or professional musicians who use them as their main instrument is surprisingly small (Michel Waisvisz, Laetitia Sonami, Nicolas Collins, Atau Tanaka, Richard Boulanger...).

- Being that live electronics and laptop music is so widespread (e.g. Cascone, 2000) it is symptomatic and frustrating that so many performers prefer to still rely on the mouse, or at the most, on generic and dull midi fader boxes.

- Commercially available new instruments are scarce and hardly imaginative and ground-breaking (e.g. Korg KAOSS Pad ${ }^{8}$ ).

- A new standard electronic instrument is yet to arrive.

\footnotetext{
${ }^{8}$ The KP2 KAOSS Pad, http://www.korg.com
} 
In effect, not any recent electronic instrument has attained the reduced popularity of the Theremin or the Ondes Marthenot, invented respectively in 1920 and 1928 (Battier, 2000). Successful new instruments exist, but they are not digital, not even electronic. The latest winner, the turntable, became a real musical instrument in the early eighties, when it started being played in a radically unorthodox and unexpected manner. It has since then developed its own musical culture, techniques and virtuosi (e.g. Poschardt, 1995; Shapiro, 1999; Hansen, 2002). The fact that so many digital turntables simulators do already exist (Kato et al., 2000; Andersen, 2003), some of them like Stanton's Final Scratch ${ }^{9}$, even quite successful commercially, gives us as many clues on the turntable wealth, as it does on the new instruments design sterility. And if commercial success is a complex subject that we may better obviate, all the previous complaints can still be summarized in one critical sentence:

Many new instruments are being invented. Too little striking music is being made with them.

\subsection{Summary}

For Leonello Tarabella the fact that it is not easy to define how to use the computer as an instrument the way it can be done with traditional instruments, is a clear proof that we still are at the "Stone Age" of computer music live performance (Barbosa, 2001). While standardization may not be a must (maybe not even desirable), highly idiosyncratic instruments which are often only used by their respective creators may not be the best sign or strategy for a serious evolution in this field.

We need useful, playable, thought-provoking enjoyable instruments, capable of interesting, surprising, enjoyable music. How can we create these "good" instruments? What is a good music instrument, anyway? Are there instruments better than others? While it is true that each culture and epoch has praised several music instruments over others ${ }^{10}$, some instruments are indeed more powerful, flexible or versatile than others. Some are vocationally generic or all-purpose while others are highly specialized ${ }^{11}$. Some take years to master, while others can be played by amateurs or even by complete novices. Some become toys after ten minutes (or two hours), while some [good ones] manage to capture our attention and squeeze our energy for decades.

Music instruments can be indeed categorized with many attributes; they can be evaluated according to many parameters, which make comparisons between different instruments meaningless unless we clearly specified the aspects involved in the evaluation. We want to discover and discuss musical instruments properties or attributes that can be relevant and helpful for the design of new instruments. Prior to find, define

\footnotetext{
${ }^{9}$ http://www.finalscratch.com

${ }^{10}$ In western culture, for example, favoritism has switched several times between strings and winds for varied religious and philosophical reasons. While Greeks clearly preferred string instruments, Roman writers claimed the superiority of winds because their imitation of the sound of the human voice (Cassiodorus, 1980). The preference shifted back to the strings between the $\mathrm{VII}^{\text {th }}$ and XVI ${ }^{\text {th }}$ centuries, apparently due to Biblical considerations.

${ }^{11}$ Both concepts will be thoroughly discussed in section 4.5.
} 
and dig into some of these attributes (which will be done in the last section of this article), we will make a quick survey on the ways musical instruments have been classified and categorized through history and cultures, and on how more modern classifications and bodies of knowledge relate to current new instrument design, practice and research.

\section{Musical Instruments: Taxonomies and Bodies of Knowledge}

\subsection{The Westerner Organology Quick Tour}

With few exceptions, all cultures around the world have developed their own formal or informal ways of classifying musical instruments, based on different criteria, ideas or beliefs, related in turn with different visions about the nature of music, science, art, knowledge, or even the cosmos (Kartomi, 1990).

Like it happens in many other disciplines, since the times of Plato and Aristotle, Western organology has followed almost always a taxonomical, tree based downward classification approach (Mayr, 1982; Kartomi, 1990). Westerners, who tend to classify as "logically" as possible, have organized musical instruments employing criteria biased towards acoustic and morphological principles (wind, string, percussion). These were the basic principles first already applied by Plato and Aristotle, and progressively refined by Boethius (c.470-c.524), Gioseffo Zarlino [1570-1590] or Michael Praetorius (Praetorius, 1980) among many others. In that sense, there is not an essential rupture between the Renaissance models of Zarlino and Praetorius and the taxonomy proposed by Hornbostel and Sachs at the beginning of the $\mathrm{XX}^{\text {th }}$ century, which is still widely used by musicologists and museologists. We will mot discuss here about the imperfections and flaws of these taxonomies; no classification can pretend to be perfect and even their authors did recognize the contradictions between the systems they propose and reality (Hornbostel \& Sachs, 1961). The essential aspect for us, is that these taxonomies are based on the mode of sound excitation and on the nature of the sounding body, concepts which can be of little help for new digital sounding instruments! The matter is not to deny Sachs' classification because $\mathfrak{t}$ is obsolete, as it works considerably well with acoustic instruments ${ }^{12}$. The key point is that the Eurocentric vision of musical instruments adds little light to the conception of digital instruments.

\subsection{Sound Oriented Taxonomies and Control Studies}

The use of two or more simultaneous (and not necessarily acoustical or morphological) parameters has been common in many other cultures classifications. The music

\footnotetext{
${ }^{12}$ Sachs first added electrophones as a new category (with three subcategories) in 1940. All current digital sound synthesis systems would be included in that category, although not sound processing systems, because "they do no produce their own sound" (!). Digital sound processing did obviously not exist in the 40s, but Sachs did explicitly not consider as electrophones, instruments such as the electric guitar, in which electricity is only used for amplifying an existing sound (Sachs, 1940; Kartomi, 1990).
} 
instrument classification of the T'boli culture of the southern Philippines for example, employs categories such as "solo" vs. "ensemble" or "gentle" vs. "strong" musical style, as shown in Table 2. But it is not until the late twentieth-century that western organologists have shown the desire to study instruments in all their complexity and shifted subsequently toward multifaceted classification (Kartomi, 1990).

\begin{tabular}{|c|c|c|c|c|}
\hline & \multicolumn{2}{|c|}{ Solo instruments } & \multirow{2}{*}{ Ensembles } & \\
\hline $\begin{array}{l}\text { NAWA } \\
\text { "played with the breath" }\end{array}$ & $\begin{array}{l}\text { FEU } \\
\text { KEYOTUT } \\
\text { FLENDEG } \\
\text { S'LOLI } \\
\text { KUMBING }\end{array}$ & $\begin{array}{l}\text { reed winds } \\
\text { flutes } \\
\text { " } \\
\text { jew's harp }\end{array}$ & & \multirow{2}{*}{$\begin{array}{l}\text { LEMNOY } \\
\text { "gentle musical style" }\end{array}$} \\
\hline $\begin{array}{l}\text { T'DUK } \\
\text { "played with the fingers" }\end{array}$ & $\begin{array}{l}\text { DWEGEY } \\
\text { S'LUDOY } \\
\text { HEGELUNG }\end{array}$ & $\begin{array}{l}\text { fiddle } \\
\text { zither } \\
\text { lute }\end{array}$ & $\begin{array}{ll}\text { SETAN'G } & \text { zither with two players } \\
\text { SEGUYN } & \text { lute and fiddle }\end{array}$ & \\
\hline $\begin{array}{c}\text { TEMBOL } \\
\text { "played by beating" }\end{array}$ & \begin{tabular}{|l} 
KLUTANG \\
S'LAGI SOTU \\
KLINTANG \\
T'NONGGONG SOTU \\
MEDEK DOL
\end{tabular} & $\begin{array}{l}\text { percussion beam } \\
\text { gongs } \\
\text { gong chime } \\
\text { drum } \\
\text { stamping trough }\end{array}$ & SEBELANG gongs, drums, sticks and chimes & $\begin{array}{l}\text { MEGEL } \\
\text { "strong musical style" }\end{array}$ \\
\hline
\end{tabular}

Table 2. Three-dimensional paradigm of T'boli instruments and ensembles (from Kartomi, 1990)

If morphological and acoustical taxonomies are of little interest for the new instrument researcher or designer, classifications based on the sound possibilities of the instruments, such as the quick view listed in Table 1 seems a more promising approach. As mentioned before, back in the $\mathrm{XVI}^{\text {th }}$ century, Praetorius had already classified instruments according to quantitative characters like how long the instrument can hold a tone and whether its tuning is fixed or not, or whether it can be made to produce all the tones and voices of a piece or only some or only one voice at a time. This approach was retaken by the German musicologist Kurt Reinhard in 1960 who as an alternative to Sachs taxonomy proposed an alternative multidimensional classification more useful for the study of musical style and the history of performance practice, based on the sonic possibilities of the instruments, very close to the ones described in Table 1 (Reinhard, 1960). Reinhard distinguishes between monophonic and polyphonic instruments, instruments with "tones that die away" vs. instruments "with continuous tones", or instruments with continuous, discrete or fixed pitch, etc ${ }^{13}$.

During the last decade, many computer music researchers, of which we will only mention a few, have studied music instruments' sound control and player-instrument communication starting from comparable sonic frameworks, trying essentially to divide the control problem into separable sound aspects and separated gestures.

(Pressing, 1990) studies and compares the sound control issues on a violin and on a standard MIDI keyboard (keyboard + wheels + pedals) using no less than ten dimensions. (Vertegaal \& Eaglestone, 1996) evaluate timbre navigation using different input devices. (Levitin et al., 2002) evaluate separately the different control aspects and actions that musicians take at the beginning, at the middle and at the end of a musical event. (Wanderley, 2001) proposes a basic gestures' taxonomy and evaluates how these gestures better relate to different sound control tasks. Wanderley, Orio (Wanderley \&

\footnotetext{
${ }^{13}$ Reinhard recommended retaining Hornbostel and Sachs' scheme when a morphological taxonomy was best suited.
} 
Orio, 2002) and other researchers such as (Rubine, 1991) have done a great job bringing general Engineering Psychology (e.g. Fitts, 1951; Fitts \& Posner, 1967), and Human Computer Interaction (HCI) knowledge and research to the particular domain of sound control.

The connection between gestural parameters (input) and sound control parameters or audible results (output) is called mapping. The most direct kind of mapping, which associates each single sound control parameter (e.g. pitch, amplitude, etc.) with an independent control dimension, has proved to be musically unsatisfying, exhibiting a toy-like characteristic that does not allow for the development of virtuosity. More complex mappings, which depending on the type of relation between inputs and synthesis parameters are usually classified as one-to-many, many-to-one or many-tomany, have proven to be more musically useful and interesting (Hunt, 1999; Hunt, Wanderley \& Kirk, 2000).

Most of this research has been carried out with standard input devices such as mice, sliders, joysticks, graphic tablets or, in lesser cases, specific existing music controllers like Yamaha's WX7 MIDI wind controller (Wanderley, 2001). With few exceptions such as the SensOrg Cyberinstrument (Ungvary \& Vertegaal, 1999), a modular and highly configurable assembly of input/output hardware devices, which seeks optimal musical expression by providing a building-blocks controller design approach, or the Stick, a music controller currently being played by Michael Brook and Laurie Anderson (Adams et al., 1996, 1999; Smith et al., 2000; Cutler et al., 2000), the primary aim of these systematic research has not been to produce any real music instrument.

What these studies have also suggested is that, even in the simplest environment, such as a one-to-one relationship between a physical parameter and a sound control parameter, the choice of the input device is still critical. A horizontal slider, a rotary knob, a pressure sensor that measures the pressure of one finger, an accelerometer which can measure tilt and respond to rapid movements, a sonar or an infrared system that can detect the distance between two points... each have their idiosyncratic properties, each can become a good or a bad choice, depending on the context, the parameter to control or the performer who will be using it (Ryan, 1991).

\subsection{Classifying Controllers}

If we omit the "array of switches" commonly known as music keyboard, music controllers are fairly recent, especially when compared to music instruments. As we mentioned earlier, the advent of MIDI brought, at east in the experimental field, the burgeoning of alternate music controllers. Commercially available controllers on their side, with honorable exceptions like the quasi epic work of Donald Buchla ${ }^{14}$ have mostly focused on constructing MIDI controller versions of traditional instruments. In that sense, many studies and classifications such as (Wanderley, 2001) distinguish between (a) instrument-like-controllers, (b) extended controllers and (c) alternate

\footnotetext{
${ }^{14}$ Buchla and associates' Homepage: $\underline{\text { http://www.buchla.com }}$
} 
controllers, the first two categories usually profiting from existing playing techniques ${ }^{15}$. In the first category, not only keyboards, but virtually all traditional instruments, clarinets or saxophones, trumpets, guitars, violins, drums, xylophones, accordions, etc. have been reconceived as MIDI controllers. While their control capabilities are always reduced when compared to their acoustic ancestors, they offer as a counterpart, an expanded, unlimited sound palette.

The second category, extended controllers includes traditional instruments (which sometimes can be even played "unplugged") with the add-on of extra sensors that afford additional playing nuances or techniques and in consequence, supplementary sound or music control possibilities (Machover, 1992; Wanderley, 2001). In that sense Wanderley studies and proposes the use of ancillary gestures (i.e. gestures common to one instrument playing technique that do not have any real sound effect or consequence, but that probably carry a semantic musical connotation) to be used as additional control sources. Although several extended controllers have been constructed to measure (e.g. for virtuosi such as YoYoMa or Winton Marsalis...) none of them is being played on a regular basis; none of them has managed to dethrone its traditional model.

When it comes to the "others", the alternate controllers not easily includable in any previous model, different authors tend to classify them according to slightly different principles. For a technical classification, based on sensing technologies (as well as on the types of muscle movements involved) readers can refer to (Bongers, 2000). Joseph Paradiso may be considered as one of the world main experts in sensing and field technologies for controllers' design, but his classification (Paradiso, 1997) favors the performer's point of view and not the constructor's approach, classifying controllers according to how they are used or weared, instead of considering how they internally work or how they can be build. Some of the new categories he proposes are batons, non contact gesture sensing (which includes Theremins, ultrasound, computer vision, etc.) and wearables (including gloves and biosignal detectors). Many other authors (e.g. Buxton, 1987; Card et al., 1991; Wanderley \& Orio, 2002) propose taxonomies of input devices that are based on HCI methodologies, considering aspects like the physical variables being sensed (position, motion, pressure, etc.), their degrees of freedom and their resolution. From the usability and the performance point of view, (Rubine \& McAvinney, 1990) constitutes a good introduction to the discussion of some of the current sensing technologies' temporal, spatial and dynamic resolutions (although some of the values shown may have improved in recent years).

\section{Playing with Space}

The world caught up with Leon Theremin in the 1960s and 1970s when several composers rediscovered the exploration of movement to create electronic music. Of particular note is Variations V (1965), a collaborative work featuring music by John Cage and choreography by Merce Cunningham, with a system designed by Gordon Mumma and David Tudor to derive sounds from the movements of dancers, who produced music based on their proximity to several electronic

\footnotetext{
${ }^{15}$ Wanderley also introduces Instrument-inspired controllers as a subcategory of instrument-likecontrollers, relating to instruments that may "look like" the original, but can also use completely new playing techniques.
} 
sensors placed on stage. Thus, the entire floor was transformed into a musical instrument responsive to movement throughout the space (Winkler, 1995,

Nyman, 1980).

Independently of the appearance or of the physical principles involved in these controllers (e.g. batons, gloves or other wearable, or non-wearable and invisible devices that track body positions and movements), a wider category that historically starts eighty years ago with the Theremin, the first instrument to be played without physical touch, could be considered; that of gestural interfaces, which includes all controllers that allows to play with and in space ${ }^{16}$. In that sense, two additional subcategories can naturally emerge: systems that allow to play with the hands (either naked or fitted with gloves, batons, etc.) like it is the case of the Theremin, and systems that allow to play with the whole body, like dancers do. The reasons for this division are merely anthropocentric; they are not biased by technological issues and are not necessarily dichotomical (some controllers or technologies can perfectly work on both domains). Researchers like Dean Rubine (Rubine \& McAvinney, 1990), Axel Mulder (Mulder, 1994, 1998), Sidney Fels (Fels, 1994) or Teresa Marrin (Marrin, 2000) have concentrated more on the first issue, while others such as Todd Winkler (Winkler, 1995), Antonio Camurri (Camurri et al., 2000) or Roberto Morales-Manzanares (Morales-Manzanares et al., 2001), have focused more on the second one. In any case, it is a matter of how to extract, analyse and map the information of the position and the movements of (part of) the human body in ways that can make the most musical sense. It is with no doubt a complex area of research in which musculo-skeletal, neuro-motor and symbolic levels have to be all addressed.

\subsection{Interactive Music Systems}

Interactive computer music systems are those whose behavior changes in response to musical input. Such responsiveness allows these systems to participate en live performances, of both notated and improvised music (Rowe, 1993).

Until now we have only considered output devices that, like traditional instruments, follow the one gesture to one acoustic event paradigm. If the mapping of performer actions to sound synthesis parameters is already generally considered as a few-to-many relationship (Lee et al., 1991; Hunt et al., 2000), what does happen when we consider as mentioned before- instruments also capable of macrostructural control? If systematic research already showed to be quite difficult, systematic research turns now to be essentially impossible.

It is also hard to provide a taxonomical view that covers of all the interactive music systems or intelligent instruments possibilities. Trying to classify them with the help of categories from traditional ways of making music, could lead to several metaphors such as, playing a musical instrument, conducting an orchestra, playing together (ensemble) with a machine or acting as a one-man band (Pressing, 1990). In order to recognize similarities between different interactive music systems, and to be able to identify the

\footnotetext{
${ }^{16}$ Although the Theremin is a whole instrument, in which the output sound process cannot be separated from the input gestures, and thus cannot be considered as a mere controller.
} 
relations between new sys tems and their predecessors, Robert Rowe proposes a more detailed three-dimensional classification (Rowe, 1993).

- The first dimension distinguishes between score-driven systems (which tend to use predetermined event collections or stored music fragments) vs. performancedriven systems.

- The second dimension considers response methods as being transformative, generative or repetitive.

- The third dimension distinguishes between the instrument and the player paradigms, the first tending to sound more a as solo and the former as a duet (in the case of a single human playing with the system).

The taxonomy has severe pitfalls (e.g. the three dimensions are severely correlated and while his author claims that the descriptors he uses are only points in the extreme s along the continuum of possibilities for a given dimension, the truth is that this continuum is not always easily conceivable), but it is still considered today as the common conceptual framework in interactive music systems. As it is impossible to seriously discuss in a brief paragraph all of its connotations and implications, we will concentrate on its third axis, based on the instrument-player opposition. For further extensions of this taxonomy the reader may also refer to (Winkler, 1998) and (Rowe, 2001), while (Casserley, 2000 $\&$ Paine, 2002) propose interesting discussions and criticisms to this Rowe-Winkler model.

When Rowe defines the instrument paradigm (as one of the extremes of the third dimension) he is taking the traditional instrument (one gesture to one acoustic event) as a conceptual model; for the remaining categories he does not consider interactive music systems as being necessarily music instruments. It is our belief that any system capable of producing music and of being controlled in eal-time (i.e. being played) could be considered an instrument. The key point lies in the concept of "control".

As exposed in (Pressing, 1990), the desirability of literalness of control is an implicit or explicit design principle in the instruments of every known musical culture. But it is also true that some interactive systems have been envisioned by their authors as behaving in an essentially "uncontrollable" way. Computer musician, trombone player and jazz improviser George Lewis, conceives for example its Voyager system, a computer program that analyzes aspects of a human improvisor's performance in real time and uses that analysis to guide an automatic composition program, as an entity that generates complex responses to the musician's playing, with an independent behavior that arises from its own internal processes (Lewis, 2000). Following Rowe's classification, Voyager functions as an extreme example of the "player" paradigm (i.e. at the opposite extreme of the instrument-player axis. For Lewis, the computer system does not function as an instrument to be controlled by a performer, but as multiple parallel streams of music generation, emanating from both the computers and the humans - a nonhierarchical, improvisational, subject-subject model of discourse, rather than a stimulus/ response setup. It is not anecdotic that Lewis' program does not posses any real-time parameter control or inputs (i.e. no sliders, no buttons...), other than the audio being played by the human performer.

Lewis' Voyager is truly the case of a "player" that needs not being considered as an "instrument", but we believe that to many others systems that could also be included in 
Rowe's "player" category, some instrumentational approach would not hurt. Rowe, especially concerned with interactive systems' unlimited output possibilities, does not discuss about any control or input issue. He considers what these systems can do, but he is not preoccupied on how they are used. With few exceptions, this is the current trend in an area (music interactive systems) that seems already too broad by its own.

\subsection{Summary}

Sound control, ergonomics, interface design, interactive music systems... In this third section we have rapidly described different approaches from which researchers are facing instruments' design. Low-level and focused research that tries to solve independent parts of the problem is clearly essential for any real progression in this field, but it is also clearly insufficient. Integral studies and approaches, which consider not only ergo nomic but also psychological, philosophical and above all, musical issues, even if non-systematic by definition, are also needed.

The last part of this article tends to conceive instruments as entities with which players establish dynamic relations. We will always be implicitly considering the wider concept of instrument we have proposed: any device used to produce any music, transforming in real-time (i.e. by being played) the actions of one or more performers ${ }^{17}$ into sound events. We will not enter into any detail concerning the inputs neither the outputs; we will not be distinguishing, for instance, between playing with sound or with form, or with both at the same time (Jordà, 2001, 2002a); anything could be also applied to traditional acoustic instruments. To study with unified criteria these instruments-to-be members of a universe so diverse in which physical model based synthesizers coexist with melodic fractal generators or mixing and looping tools- seems indeed a hard task. We also believe if can be a fruitful and clarifying one. We will not tell what computerbased instruments should be or how "computers should be played", but we will try to define and study concepts such as efficiency, apprenticeship, learning curve, path to virtuosity or expressivity; concepts that may help at describing and identifying the dynamic relations existing between players and the ir instruments. We will discuss these properties trying to find what may constitute the essential needs for different types of musicians, which can be the elements that may turn a sound toy into a tool for musical expression, and this into an instrument for virtuosi.

The reader should not be however too naively impressed by some of the following pseudo-mathematical formulas. They are not meant to be magical recipes; no fixed design or construction rules for delivering better new instruments. They should be taken as the guidelines that help to conduct some of my personal intuitions and beliefs; ideas that rise after trying to extract some order out of years trying to design new instruments, listening and watching people playing music with them and, essentially, improvising with them myself (Jordà, 2001, 2002a; Feller, 2002; Jordà, 2003a).

\footnotetext{
${ }^{17}$ Although we will be referring to the player and instrument concepts, mostly in singular, everything could be directly applied to collaborative and collective instruments (e.g. Jordà, 2002a; Blaine \& Fels, 2003).
} 


\section{The Instrument and the Player}

\subsection{What is a good musical instrument, then?}

We know how to make musical instruments that sound great, but can we make them easier to learn, better adapted to each individual's gestural expression preferences and movement capabilities?" (Mulder $\left.{ }^{18}\right)$.

At the opening of the conference in Dublin, keynote speaker Tod Machover launched several questions of which I here retain two: "How do we create controls and interactions that feel inevitable to expert and amateur users?", "how do we create interactive situations that stimulate rather than placate, leading the participant beyond the surface and into thoughtful consideration of rich, expressive, meaningful experiences?". According to him, the last two decades have seen successful designs of controllers capable of virtuosity and subtlety, and also of controllers that "hook" novice users, but in this last case, very few systems have been nourishing as well, capable of encouraging deeper exploration and continued discovery and creativity (Machover, 2002). And this is a feeling shared by many computer music instrument designers and computer music performers.

Our design criteria include initial ease of use coupled with a long term potential for virtuosity, minimal and low variance latency, and clear and simple strategies for programming the relationship between gesture and musical result (Wessel \& Right, 2002).

\section{Nothing is perfect. Anything can get better (and worst!)}

Humans have been playing music for thousands of years, but sudden innovations in human performance, even in millenary experienced areas seem indeed possible. Good examples can be found on sports, by far the most advanced and sophisticated area concerned with human responsiveness and techniques: the crawl stroke swimming style and the Fosbury jumping are $\mathrm{XIX}^{\text {th }}$ and $\mathrm{XX}^{\text {th }}$ centuries techniques, invented from scratch after millennia of humans swimming ${ }^{19}$ and jumping!

Acoustic musical instruments as we know them now are also the fruit of centuries or even millennia of evolution; they have settled into canonical forms. But as we will see, that does not necessarily imply that these instruments are perfect or that they all excel at whatever parameter we evaluate.

\subsection{Introducing the Odd Quartet}

\footnotetext{
18 Mulder, A. Designing musical instruments that performers can handle [On-line]. Available: http://www.cs.sfu.ca/people/ResearchStaff/ amulder/personal/vmi/vmi.html

19 The crawl stroke was in fact developed around the turn of XIX ${ }^{\text {th }}$ century, by English and Australian swimmers who copied techniques used by native peoples in Australia and Ceylon.
} 
For the following experiments we will pick four instruments, the kazoo, the kalimba, the piano and the violin (an odd quartet for sure!), and try to figure out what it is that makes some musical instruments more enjoyable than others.

\section{Balance (Challenge, Frustration, Boredom...)}

Many traditional instruments are quite frustrating for the beginner. The violin, for instance, can hardly be taken as a toy (whereas even the piano could). Some instruments are definitely harder to play than others. Wind instruments, for instance, are frequently hard to blow; the absolute novice cannot produce any controllable sound (there are exceptions, such as the recorder family). Fretless string instruments are impossible, for a novice, to play in tune. And with fretted ones, if multistringed, the novice may have a hard way deciding what string to use between all the possible options, in order to obtain a desired pitch. Keyboard instruments seem more direct: they produce sound easily, and there is a bijective relation between pitches and keys. And while piano music can be the most complex music (and therefore the hardest to play) it can also be quite simple though, and an absolute non-musician can still improvise some beautiful piano music.

I have being developing computer-based interactive music systems since 1989. Some of them like PITEL or Afasia were conceived for trained musicians or even for specific performers (Jordà, 1991, 2002b) while others, like Epizoo, were to be controlled by members of an audience in public performances (Jordà, 1996). The demands for the two genres are usually very different. Complicated tools, which offer great freedom, can be built for the first group, while the second group demands simple but appealing tools that -while giving their users the feeling of control and interaction must produce "satisfactory" outputs (e.g. Ulyate \& Bianciardi, 2002). These two classes are often mutually exclusive. Professional musicians become easily bored with the "popular" tool, while the casual user may get lost with the sophisticated one. Good music instruments must strike the right balance between challenge, frustration and boredom: devices that are too simple tend not to provide rich experiences, and devices that are too complex alienate the user before their richness can be extracted from them (Levitin and Adams, 1998). The kazoo is easy to master, but its possibilities are quickly exhausted. We should be able to design well balanced instruments that can appeal to both professionals and dilettanti; instruments that like the piano, can offer a low entry fee with no ceiling on virtuosity (Wessel \& Wright, 2002).

\section{Playability, Progression and Learnability}

In our odd quartet, we will discard the violin because it needs a will of iron (not the best feature for initiating digital instruments proselytism!). We also discard the kazoo because it cannot go that far. With the remaining two instruments things get more delicate. The piano definitely offers no ceiling on virtuosity, while a Westerner may lodge some doubts about the kalimba. But what happens during the ir learning path? An absolute non-musician can still improvise some beautiful piano music, we argued, but still, the piano is a really intimidating instrument and the kalimba is not. Kalimbas have few notes (all of them "correct" ones). Its shape and construction intuitively invites anyone, as Donald Norman's scissors (Norman 1990), to play it the correct way: with the thumbs. And once you take a kalimba in your hands the decision about what thumb to use for a given note is almost direct (obviously, not the case of the piano!). It is true that the kalimba has little dynamic control, but it cannot be considered as a restricted 
instrument, at least when compared with the "average public interactive sound installation". It is a well designed instrument "for all ages" (Jordà, 2002c). At the risk of sounding too hazardous I will suggest that, at least at their first stage, the kalimba is a more efficient instrument than the piano.

\subsection{The learning curve}

The learning curve is a widespread concept, systematically used in all areas that involve any aspect of apprenticeship, and vaguely interpreted as the graphical representation of a progress in learning. The term is also widely used in music education and in new controllers studies (improve the learning curve, steeper learning curve, better learning curve...) (e.g. Rovan et al, 1997; Wanderley \& Orio, 2002; Pressing, 1990; Vertegaal et al., 1996; Blaine \& Fels, 2003). But was does "learning curve" exactly mean? Is there a way of measuring it?

Learning curve: A graph showing some measure of the cost of performing some action against the number of times it has been performed. The term probably entered engineering via the aircraft industry in the 1930s, where it was used to describe plots showing the cost of making some particular design of aeroplane against the number of units made.

The term is also used in psychology to mean a graph showing some measure of something learned against the number of trials. The psychology graphs normally slope upwards whereas the manufacturing ones normally slope downward but they are both usually steep to start with and then level out.

Marketroids often misuse the term to mean the amount of time it takes to learn to use something ("reduce the learning curve") or the ease of learning it ("easy learning curve"). The phrase "steep learning curve" is sometimes used incorrectly to mean "hard to learn" whereas of course it implies rapid learning. (From hyperdictionary ${ }^{20}, 2003$ )

This definition does not fit too well within our musical scheme. First because we do not want to evaluate the cost of a given fixed action, as we intuitively assume that as the learning curve evolves, the musical actions undertaken can be more and more ambitious. Second, even if we considered variable, increasingly complex actions, how could we evaluate their cost? Since we are considering real-time scheduled activities, these can hardly be measured in time (i.e. the time it takes to accomplish the task), as the common practice is.

In a musical context, (Levitin, 2002) describes the learning curve as the amount of time it takes to a novice to gain enough skill with an instrument so the experience of playing it becomes rewarding. Essential as it is, this concept does obviously not define a curve but a (very important) point of this curve, we could call the 'rewarding point'. (Wanderley \& Orio, 2002) when studying the usability of different controllers, define learnability as the time needed to learn how to control a performance with a given controller. A time, they suggest, longer than ten years for most traditional instruments

20 http://www.hyperdictionary.com/ 
(Lehman, 1997). This defines another important point of the curve that we could call the 'mastering point'.

We intuitively grasp the learning curve concept; we know it can tell us a lot of information about the relation between a player and an instrument, on how the relation starts and evolves, but we do not know how to clearly define this concept, much less how to evaluate it. Could we, in order to compare different instruments, compare their learning curves? We can compare their learning curves shapes and tendencies, but we cannot evaluate their asymptotes or their inflexion points since we do not know the ordinates absolute values, not even what they represent. A serious attempt at defining the music instruments learning curve concept falls outside the pretension of this article, but that will not dismiss us from trying to manage the concept more intuitively.

The piano has a steeper (i.e. better!) learning curve that the violin. The kalimba has an even steeper learning curve, although its asymptote may remain lower. The learning curve of the kazoo is almost a straight horizontal line, very low when compared to the other three asymptotes (since the abscissa of its 'mastering point' is very close to the time origin, and the ordinate is also close to cero given the reduced capabilities of the instrument). All these approximate criteria are suggested in Figure 1.

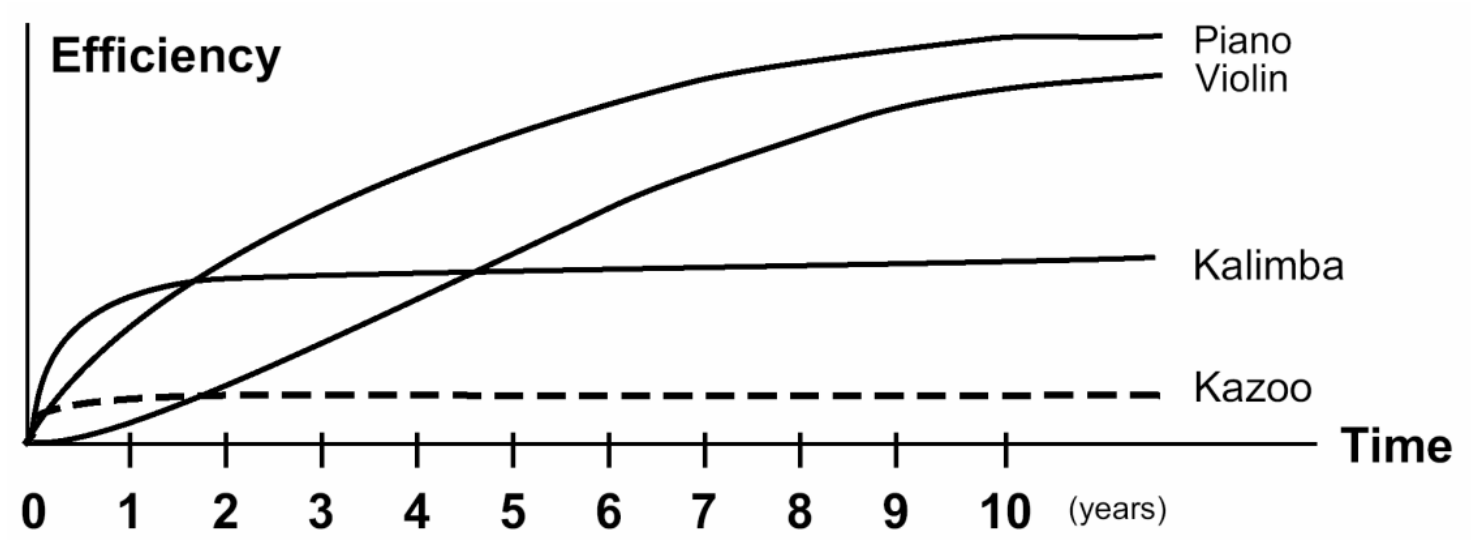

Figure 1. Approximate learning curve for the (a) kazoo, (b) kalimba, (c) piano and (d) violin, within a period of 10 years

\subsection{Efficiency of a Music Instrument}

We ended up section 4.2 claiming that the kalimba could be considered a more efficient instrument than the piano, without saying a word about we should consider by efficiency of a music instrument. In engineering, the term 'efficiency' is commonly understood as the ratio of useful energy output to energy input. (Timoshenko \& Young, 1937) define for example the efficiency of a machine as the ratio of the useful work performed to the total energy expended.

We ended up section 4.2 claiming that the kalimba could be considered a more efficient instrument than the piano, without saying a word about what we should consider by efficiency of a music instrument (a concept that to my knowledge has never been used outside of the acoustic domain) (Jordà, 2003b). In engineering, the term 'efficiency' is commonly understood as the ratio of useful energy output to energy input. Timoshenko 
and Young [20] define for example the efficiency of a machine, as the ratio of the useful work performed to the total energy expended.

$$
\text { Efficiency }=\frac{\text { Output }}{\text { Input }}
$$

(Macleod et al., 1997) studied efficiency in a Human-Computer-Interaction context (HCI), affirming that in a system in which a human is interacting with a computer, the effectiveness with which the user and the computer working together successfully complete a task, is a measure of the useful work performed, or the output. The nature of the output being in this case quite different from the input, no adimensional ratio is possible. According to the authors, this does not imply however that we are not able to express the efficiency of task performance numerically; it simply means that efficiency measures will be now expressed in some units. After which they quantify the amount of effort input from a user's viewpoint, as the mental/physical effort required to complete it:

$$
\text { HumanEfficiency }=\frac{\text { Effectiveness }}{\text { Effort }}
$$

Our context is not that different. We will start with a quite similar and simple quotient (with quite fuzzy terms, we must admit!) that will allow us to roughly estimate the efficiency of a music instrument according to the following ratio:

$$
\text { MusicInstrumentEfficiency }=\frac{\text { MusicalOutputComplexity }}{\text { ControlInputComplexity }}
$$

- The Musical output complexity will depend on all the available sound aspects and variability properties, as those exposed in Table 1. It should consider for example, both the microsonic richness of the violin and the mid-sonic richness of the piano), plus all the potential macrostructural complexities that could be managed by any kind of intelligent instrument. This term can be related to the musical range defined by (Blaine \& Fels, 2003) and to the expressive range described by (Settel \& Lippe, 2003) ${ }^{21}$.

- The Control input complexity, depends on factors like the degrees of freedom of control, the correlation between these controls and the convenience of the applied mapping. It could also be linked to (Orio, 1999) explorability, which is defined as the number of different gestures and gestural nuances that can be applied and recognized, and which related to controller features such as precision and range and also to the mapping strategy.

Under different names and with slightly diverse approaches, both concepts are clearly being studied elsewhere. In particular, a thorough understanding of the control input complexity would lead us to areas associated with engineering psychology, closely

\footnotetext{
${ }^{21}$ For these authors, the expressive range, which determines the musical potential of an instrument, can be divided into "dynamic, timbre and pitch range" and responsiveness or instrumental depth
} 
related to the work of Rubine or Wanderley and other researchers mentioned in section 3.2 , and completely out of the scope of this exposition. The point is that the concept of 'music instrument efficiency' - a term that to our knowledge, has never been used outside of the acoustic domain- as vague as it still remains, could constitute, when plotted vs. studying or practicing time, a better-than-nothing first approximation for describing the learning curve. As a performer learns to play an instrument, the attainable output complexity increases (while staying below the theoretically limited maximum complexity the instrument is capable of outputting). Meantime, control complexity can also augment, which explains why after a certain period of time, efficiency does not necessarily follow a clear ascending curve. It happens in fact, that after a long period of studying (e.g. 10 years), the input cost needed for maintaining a high-valued output becomes so elevated that many professionals-to-be decide to abandon their instrument. This new approach to efficiency will also constitute an essential guideline for the remainder of this exposition.

\section{Playing [with] Music?}

Bongers and Wanderley among others, describe interaction in sound installations as a context where one or more persons' actions are sensed to provide input values for an audio generating system. They state that since users do not know precisely how to interact and that no special skills are required, the primary goal of the interaction is not necessarily the expression of some information (Bongers, 2000; Wanderley \& Orio, 2002). While the se conditions are generally true, we should not forget the cases in which users are allowed to take more time to investigate and to learn. Interactive hardware-based music toys as well as software music games, either off-line or on-line, could perfectly illustrate this category.

In ancient Greece, the kithara was usually played by professional musicians as it required some kind of exhibitionism and virtuosity that could be considered vulgar and not adequate for free-born men. The lyra instead, was commonly played by amateurs, and was, according to Plato, a well suited instrument for free citizen (WinningtonIngram, 1980). Before the advent of the radio and recorded music, families who could afford it, tend to have an instrument at home (often a piano) and some of the family members were enough talented to become "animated interactive CD players" every evening.

“...it becomes possible for more people to make more satisfying music, more enjoyably and easily, regardless of physical coordination or theoretical study, of keyboard skills or fluency with notation. This doesn't imply a dilution of musical quality. On the contrary, it frees us to go further and raises the base-level at which music making begins." (Laurie Spiegel, from Chadabe, 1997)

Considering that the best way to understand and appreciate any discipline, whether artistic or not, and music is no exception, is by doing and being part of it, we do seriously need more efficient instruments in which the basic principles of operation are easy to deduce, while, at the same time, sophisticated expressions are possible and mastery is progressively attainable; instruments that can bring new sophisticated possibilities and the joy of realtime active music creation to non-trained musicians. Let us try, as Robert Rowe suggests, to develop virtual instruments that do not just play 
back music for people, but become increasingly adept at making new and engaging music with people, at all levels of technical proficiency (Rowe, 1993, p. 263).

Historically, the field of music controllers design was focused on advanced and sophisticated controllers aimed at professional musicians; the orientation has began to change in the last years, with many new controllers and instruments being designed for novice players, children or music aficionados (Blaine \& Fels, 2003). Todd Machover and M.I.T's Brain Opera and Toy Symphony ${ }^{22}$, the Jam-O-Drum and the Jam-O-World (Blaine \& Perkis, 2000; Blaine \& Forlines, 2002) or SmallFish ${ }^{23}$, are perfect examples of collective interactive sound installations that aim to explore an exploit the musicality of their audiences. Weinberg's Sequeezables and Beatbugs (Weinberg \& Gan, 2001; Weinberg \& Jennings, 2002), Cook's PhISEM Shaker (Cook, 2001), Paradiso's Musical Trinkets (Paradiso \& Hsiao, 2000), Patten's Audiopad (Patten et al., 2002), Poupyrev's Augmented Groove (Poupyrev, 2000) or Robson's varied Sound Toys (Robson, 2002) are only some examples of a long list of new music instruments that, with different levels of complexity and different potentiality, want to be simple, intuitive, playful, enjoyable but also responsive and musical. And this is only the beginning.

\section{The Art of Making Music Made Easy (?)}

The Art of Making Music Made Easy.

It's true, making music is an Art, traditionally demanding years of study and practice to be successful. -- until now! Enter the new Suzuki Omnicord. If you can read this text, you can play the Omnicord, and play it well. There's no need for lessons or years of study to play and sing your favorite songs right now! Just press a chord button and strum the SonicStrings. It's that easy. (from Suzuki's Omnichord $^{24}$ publicity)

Is this what we are talking about? Is it there where we are willing to go? Computer aid interactive music systems can have many applications; each of them perfectly licit and with its own place in the market, but it is not the aim of this article to trivialize creation. To insist on this essential point and try to make it clearer, let's augment our odd quartet with a fifth member, the CD player. It is an instrument very simple to use, yet capable of all the imaginable music variety and complexity; an instrument that allows everyone to play Répons as effectively as Boulez "playing" it with the Ensemble Intercontemporain $^{25}$ (Boulez, 1999). According to our previous formula, the CD player is probably the most efficient instrument we can imagine!

\footnotetext{
${ }^{22}$ Toy Symphony: http://www.toysymphony.org

${ }^{23}$ SmallFish:http://hosting.zkm.de/wmuench/small_fish

${ }^{24}$ Suzuki Omnichord, http://www.suzukimusic.co.uk/suzuki omnichord.htm

${ }^{25}$ Yes, we are suggesting here that an orchestra could possibly become an instrument under "the hands" of a conductor; an assertion to serious and with too many implications that should not be thrown like that without any discussion...
} 
The CD player example clearly parodies the [non-at-all]interactive music system situation Satirical as it may sound, this tricky illusion is used indeed in many of the current interactive sound installations: seeking to guarantee a complex or predefined musical output, many of these installations do not give to their interactors more than a couple of bits to play with ${ }^{26}$. We could add that this is not only frequent in sound or music installations; faked or useless interactivity is indeed the blot of contemporary Interactive Arts!

To penalize these poorly interactive situations we will introduce into our formula an additional term, we will call PerformerFreedom, in such a way that when the PerformerFreedom tends to zero so does Efficiency. An ideological correction that destroys the illusory potential of the Omnichord or the CD player.

$$
\text { MusicInstrumentEfficiency } \text { Corrected }=\frac{\text { MusicalOut putComplexity } \times \text { PerformerFreedom }}{\text { ControlInputComplexity }}
$$

How could this diversity control be evaluated? Once the $\mathrm{CD}$ is on the tray, the regular user does not have so many options: zapping tracks, fast-forward listening, changing volume. What the performer can do and the ways she can express and communicate with the instrument is taken into account in the aforementioned control input complexity. What the performer, by means of these actions, can ask the instrument to do and how these actions can affect the music, is what the diversity control tries to evaluate, which in a less simplistic case, is surely not a straightforward task.

This diversity control obviously relates to the performer not being the instrument's slave. A good instrument should not impose its music on the player. A good instrument, for example, should not be able to produce only good music. A good instrument should also be able to produce 'terribly bad' music, either at the player's will or at the player's misuse. ${ }^{27}$ Only if these conditions are sufficiently fulfilled will an instrument allow its performers to play music and not only to play with music.

\subsection{Musical instruments' output diversity}

We will try to refine the concept of instruments' musical output diversity. Several authors such as Vertegaal et al. (1996), Blaine \& Fels (2003), Nishimoto et al. (2003), and Settel \& Lippe (2003) have discussed "instrument versatility or flexibility" from slightly different points of view and criteria. In our approach, we propose to start distinguishing between three levels of diversity, which we will label micro-diversity, mid-diversity and macro-diversity. Before we continue, three clarifications will be made in order to avoid misinterpretations: (a) the different levels do not relate to diverse time scales; (b) the levels subjectively relate to music, not to sound and therefore not directly

\footnotetext{
${ }^{26}$ That does not prohibit the CD player from becoming, when played by sensitive musicians such as Yasunao Tone (Tone, 1997) or Nicolas Collins, a wonderful music instrument (Collins, 1992). And the same could be said about the Suzuki Omnichord, an incredible surprise when played by the German improviser Joker Nies (Nies).

27 Misuse should not be interpreted here with ideological, moral or esthetical connotations. What we suggest is that only when a performer is capable of relating unwanted results (effects) with the actions taken (causes) will this performer be able to learn and effectively progress.
} 
to psychoacoustical perception; ${ }^{28}$ and (c) although it may be more realistic to consider these levels as points on a continuum, we will maintain this construction for the purpose of explication.

\subsubsection{Micro-diversity (Performance nuances)}

Micro-diversity $(\mathrm{MicD})$ is the first that comes to mind. It permits subtle nuances, giving a measure of how much a performer can turn a piece into her own or, consequently, how much two performances of the same piece can differ. All professional traditional instruments offer many possible nuances and subtleties (Rubine \& McAvinney, 1990), therefore showing a high MicD. Whereas for acoustic instruments MicD is clearly linked to the richness and the fine control of sonic details, in digital instruments, MicD can also be related to any kind of fine structural variation (time, tempo, number of voices, density of the voices and relations between them) that could occur within a given piece, while keeping it recognizable.

An interesting example could be found in the turntable already introduced in section 2.5. While John Cage can be regarded as the conceptual 'inventor' of the instrument, before the development of new playing techniques by hip hop turnablists in the late 70s, the turntable MicD could be considered almost null: any new 'performance' of the same piece (i.e. the same vinyl) was almost identical to the previous ones. It is when its $\mathrm{MicD}$ raises by means of new performing techniques that the turntable finally becomes a real instrument.

\subsubsection{Mid-diversity (Performances contrasts)}

Raising the diversity threshold, one could ask how distinct two different pieces or two performances played with the same instrument can be. We will call this measure Middiversity (MidD). Most traditional instruments have a high component on this level, although percussion and instruments with fixed or indefinite pitch could be considered to have, at least for Westerner ears, lower MidDs. Putting it another way, the lower its MidD, the more an instrument will seem to be "always playing the same piece."

Many digital instruments and interactive sound installations designed for novices have indeed a very low MidD (Blaine \& Fels, 2003). A special case of very low MidD application also appears in the specialized and professional computer music domain, with so called open or interactive compositions. A highly sophisticated 'instrument' with a low MidD may be a very good interactive composition, but should not be considered as an instrument, even if it comes bundled with a hardware controller. If we try to apply again the cluster metaphor, MidD could probably indicate the 'maximum difference' two clusters could show (e.g. when hitting with our underarm the two opposed regions of the piano).

\subsubsection{Macro-diversity (Stylistic flexibility)}

Our third component, macro-diversity $(M a c D)$ indicates the flexibility and the adaptability of an instrument, its ability to be played in different contexts or music

\footnotetext{
${ }^{28}$ Rubine \& MacAvinney (1990) constitutes a good introduction to psychoacoustics and ergonomics for instrument designers. Their paper summarizes "just noticeable differences" (JNDs) for different sound control parameters, providing many of the source references as well.
} 
styles, to assume varied musical roles, its capabilities of integration with different instrumental partners. Instruments with a high $M a c D$ could be considered all-purpose, while a low $M a c D$ denotes a more specialized and less versatile instrument. In a traditional music context, $M a c D$ does not seem to be a necessary quality. Generic instruments that do fairly well in completely different musical contexts will probably hardly excel in any of them.

On the good side, generic instruments tend to be more rewarding without having to be mastered. They are probably better adapted to 'solo amateur music' than more specialized low MacD instruments, and seem better suited to autodidactic apprenticeship. The guitar is an example of a high $M a c D$, all-purpose instrument. On the opposite, the double-bass, could be considered as a more specialized, lower MacD instrument, but it still is extremely macrodiverse when compared to most of the new digital instruments. Could the cluster metaphor also be employed to illustrate MacD? One response to that question could be that a cluster-able instrument with a high MacD should probably be able to play also other things than clusters!

Some really versatile and chameleon-like instruments can behave both as generic and as highly specialized instruments. An instrument of these characteristics would show a wide space with several specially well-defined and connected subregions. This could be again the case of the guitar, or even more notably the electric guitar (guitar + amplifier + pedals). While the three-chord accompaniment guitar is the perfect generic instrument paradigm, the guitar is also able to define by itself several virtuosic styles, such as flamenco, or all the electric-blues-heavy-metal derived archetypes. In that sense, simultaneous high and low MacD instruments such as the guitar would probably make good candidates for the "desert island" instrument category (Settel \& Lippe, 2003).

If we had to choose one of the poles, chances are that absolute beginners will prefer an instrument that seems to offer more varied possibilities (high MacD), while professionals will possibly be more satisfied with an instrument with a stronger personality (low MacD). Since we are talking about instruments and styles that do possibly not yet exist, it would also seem logical that more idiosyncratic, low MacD instruments, would have more chances to give birth to new music styles, but high MacD instruments have also more chances for starting being used. ${ }^{29}$

Of the three diversities introduced, $M a c D$ seem the least crucial of all. It can be considered as a plus for 'catching candidates'. In contrast MidD seems fundamental for turning music gamers into music performers. Concerning $M i c D$, given that differences and nuances between one performance and another and between one performer and another are the essential elements that can help distinguishing and appreciating performers, MicD is indeed fundamental for turning a musician into a potential virtuoso.

\footnotetext{
29 If we consider for instance, two of the electronic music "styles" most solidly bound to specific "instruments", such as Acid House tighten to the Roland TB-303, and glitch or postdigitality (Cascone, 2000) linked with the laptop+mouse+MAX, we see that while the 303 has probable a very low MacD (and also quite low MidD), the laptop+mouse+MAX triplet seems indeed the most generic (high $M a c D$ ) imaginable instrument.
} 


\section{Instrument Diversity and Digital Instruments Corollaries: (Programming, Configurations-Setups and Improvisation)}

- An instrument must be able to play different pieces without the need of being reprogrammed; performers are not necessarily programmers ${ }^{30}$.

- Some instruments may have a "natural" high MidD; others may need to work with different configurations or setups.

- If different pieces need different setups, all these setups should not contradict the basic playability rules of the given instrument. Otherwise, we are not dealing with an instrument anymore, but with a variable controller+generator system.

- If setups are a must, the possibility to load them on the fly will promote improvisation.

\section{Improvisation}

Any musical instrument must permit improvisation (e.g. Ryan, 1991; Jordà, 2001 and 2002a; Settel \& Lippe, 2003). Both MidD improvisations (not on any precise piece) and MicD improvisations (structured improvisation on a given piece) should be allowed. While $M a c D$ does not seem so important it may facilitate free-form (and free-context) improvisation.

\subsection{Variability + Reproducibility = A path to Virtuosity?}

As we have seen, $M i c D$ variability seems an essential component towards virtuosity, it is not less true, that its opposite, "exact" reproducibility (resulting from an absolutely precise performing control) has been highly praised on Westerner music for the last half millennium. In that sense, a virtuoso-to-be may want to be able to guaranty the highest similarity on two performances of the same piece, a capability that (Wanderley \& Orio, 2002) define as controllability.

Whether a high reproducibility is important or not, depends obviously on the performer's personal tastes and goals. For Joel Chadabe, for example, the essence of intelligent instruments is precisely their ability to surprise and provoke the performer (Chadabe, 1984, 1997). To paraphrase Walter Benjamin, in the age of digital reproduction, more radical positions could licitly consider "exact" reinterpretation as being as creative and useful as "painting by numbers", although this is a debate we will not be addressing in these pages.

\section{[Non] Linearity and Control}

Non-linearity is not only present in complex digital instruments. Mappings in acoustic instruments are often multidimensional and slightly non-linear. Blowing harder on many wind instruments not only affects dynamics but also influences pitch, and it is in this control difficulties where may lay in fact the expressiveness for many acoustic instruments. As Johan Sundberg suggests, the voice organ, possibly the most expressive

\footnotetext{
30 Although some performers-programmers, using live coding techniques may perform while programming (e.g. Collins, 2002; Wang \& Cook, 2004).
} 
instrument ever, can also considered, taking into account the complex relationship between articulator movement and formant frequency changes that makes articulation/formant frequency a one-to-many system, as one of the most badly designed from an engineering point of view (Sundberg 1987).

Many traditional instruments can be driven to a state of chaotic behavior characterized by noisy, rapidly fluctuating tones. Examples could be found in the vocalized saxophone style in which vocal sounds interact directly with vibrations in the saxophone (Menzies, 2002). The use of feedback in the electric guitar, converts the guitar into an element of a complex driven system, where the timbral quality and behavior of the instrument depends on a variety of external factors; i.e., distance to the speakers, room acoustics, body position, etc. (Paradiso, 1997). Musicians explore and learn to control these additional degrees of freedom, producing the very intense, kinetic performance styles upon which much of free-jazz and rock music is based.

\section{Predictability}

Instruments with certain randomness or non-linearity cannot be absolutely predictable, making two performances always different, and as randomness and non-linearity increase, the instrument becomes less and less masterable and learnable. In that sense, non-linearity should not inhibit the performer from being able to predict the outputs related with small control changes, since that seems necessary for the development of a finely tuned skill and an expressive control.

A balance between randomness and determinism, between linear and non-linear behaviors, needs therefore to be found.

\section{Confidence}

Non-linearity should not mean uncontrol. In a good performance, the performer needs to know and trust the instrument, and be able to push it to the extremes, to bring it back and forth of these non-linearity zones. There is nothing worst in a new digital instruments performance, than the performer looking astonished at the computer screen.

Is it working? Is it not? Is it doing what it is supposed to do? Whose fault it is? Mine, who don't master the instrument yet? The sensors, maybe one cable broke? Is it the software, a bug?... Upps...the mixer was down!

And yet, it happens. It happens so much that I would venture that the feel of security frequently turns to be the essential criteria of a performance quality. When we find a performer that does not care about the monitor, when we see someone on stage capable of lovely caressing and of violently striking the instrument without any fear, chances are we are facing a memorable performance. Only when a performer is absolutely confident about the more essential element of the instrument, this performer can start making music.

And again, nothing is simple as you may have already noticed. On one side, confidence is necessary but no sufficient to guaranty virtuosity. On the other hand, virtuosity is not sufficient but neither necessary to develop expressivity. The recent history of popular 
music, take rock and blues for instance, could bring us many examples of this last statement.

\subsection{Summary}

Given the infinite possibilities that new instruments bring from all points of view (i.e. (a) ways of entering control information and the nature of this information, (b) output possibilities, and (c) the ways these input and these outputs can be linked), it makes sense to study them from conceptual and generic musical ways, considering them as abstract entities reduced to their common denominator: devices that independently of their intrinsic characteristics, may all output music when played by humans. Following this path, in this last section we have studied the dynamic relation that builds between the player and the instrument, introducing concepts such as efficiency, apprenticeship and learning curve. We have explored some music instruments generic properties such as the diversity, the variability or the reproducibility of their musical output, the linearity or non-linearity of their behavior, and tried to figure out how these aspects can bias the relation between the instrument and its player, and how they may relate to more commonly studied concepts such as expressivity or virtuosity.

All of these concepts would need much deeper and serious discussion and investigation Many additional ones should also be considered. Due to space and time limitations, we have consciously omitted all concepts dealing with the notion of time (their study is too often reduced to resolution and latency [short-scale] measurements evaluated from nonmusical engineering and motor learning approaches). We leave all that for future explorations.

\section{Conclusion}

Digital technologies are drastically redefining the notion of music instrument. This article constitutes an initial attempt at trying to study the dynamic relation that exists between a player and an instrument from new points of views, introducing some concepts that can be taken into account, and some questions that could be posed at the time of designing a new music instrument. What kind of music should the instrument be able to play? Who are we designing the instrument for? Are we constructing a music instrument or a music toy? Do we pretend to construct an instrument that can appeal to a wide range of musicians, from the perfect novice to the professional? Are we considering the different evolutionary steps of this possible relation? Are we guarantying the minimal elements that can make this instrument enjoyable from the beginning and potentially learnable? As we previously announced, no magic formulae or recipes, no design or construction rules for delivering better new instruments were to be discovered. Before constructing them, we should know what "better" means. The aim of this article is the foundation of a possible theoretical framework in which the possibilities and the diversity of music instruments as well as the possibilities and the expressive freedom of human music performers could all be evaluated; a framework that could help to figure out what the essentials needs for different types of musicians from the absolute novice to the professional or the virtuoso- may be. 


\section{Acknowledgments}

Thanks to Xavier Serra, Martin Kaltenbrunner and Günter Geiger at the MTG of Barcelona, for their support and their advises and suggestions during the writing this paper. I would also like to thank Joel Chadabe for his constructive comments. This work is in debt with many authors that have addressed comparable or complementary questions, such as (Pressing, 1990; Rubine \& McAvinney, 1990; Ryan, 1991; Chadabe, 1997; Waisvisz, 1999; Cook, 2001; Wessel \& Wright, 2002; Robson, 2002, Settel \& Lippe, 2003; or Blaine \& Fels, 2003) to name only a few. Even more influential have been the music and the performances of great computer music improvisers such as Michel Waisvisz, Nicolas Collins (Collins, 1991) or Tim Perkis (e.g Bischoff \& Perkis, $1989^{31}$ ). Last but not least, is the infinite listing of the "unplugged analog" musicians and improvisers who remain for me a permanent source of inspiration. It is all about making music, after all.

The research presented in this article has been partially funded by the EU project SemanticHIFI under the 6th Framework Programme (IST-507913).

\section{References}

- Adams, R. L., Brook, M. B., Eichenseer, J. W., \& Smith, G. M. (1996). (Levitin, D. J., Ed.). The Stick. Technical Report, IRC 1996-033. Palo Alto, CA: Interval Research Corporation.

- Adams, R. L., Brook, M. B., Eichenseer, J. W., Goldstein, M. H., \& Smith, G. M. (1999). Electronic Musical Instrument. USA: Interval Research Corp, Patent number 6,005,181.

- Andersen, T.H. (2003). Mixxx: Towards Novel DJ Interfaces. Proceedings of the 2003 Conference on New Interfaces for Musical Expression (NIME-03), 3035. Montreal.

- Barbosa, A. (2001). Overview and conclusions of the Music Interfaces Panel Session at the MOSART Workshop, Barcelona, 2001 [On-line]. Available: http://www.iua.upf.es/ abarbosa/docs/mosart-interactivity_pannel.pdf

- Battier, M. (2000). Electronic Music and Gesture. In M. M. Wanderley \&. M. Battier (Eds.), Trends in Gestural Control of Music. Paris: Ircam-Centre Pompidou.

- Bischoff, J., \& Perkis, T. (1989). Artificial Horizon. CD Audio, Artifact Recordings.

- Blaine, T., \& Fels, S. (2003). Collaborative Musical Experiences for Novices. Journal of New Music Research, 32(4), 411-428.

- Blaine, T., \& Forlines, C. (2002). Jam-O-World: Evolution of the Jam-O-drum Multi-player Musical Controller into the Jam-O-Whirl Gaming Interface.

\footnotetext{
31 "For us, composing a piece of music is like building a new instrument, an instrument whose behavior makes up the performance. We act at once as performer, composer and instrument builder, in some ways working more like sculptors than traditional musicians". Sleeve notes from Bischoff/Perkis, Artificial Horizon, Artifact Recordings 1989.
} 
Proceedings of the 2002 Conference on New Interfaces for Musical Expression (NIME-02), 17-22. Dublin.

- Blaine, T., \& Perkis, T. (2000). Jam-O-Drum, A Study in Interaction Design. Proceedings of the ACM DIS 2000 Conference. NY: ACM Press.

- Bongers, B. (1994). The Use of Active Tactile and Force Feedback in Timbre Controlling Musical Instruments. Proceedings of the 1994 International Computer Music Conference, 171-174. San Francisco, CA: International Computer Music Association.

- Bongers, B. (2000). Physical Interfaces in the Electronic Arts - Interaction Theory and Interfacing Techniques for Realtime Performance. In M. M. Wanderley \&. M. Battier (Eds.), Trends in Gestural Control of Music. Paris: Ircam-Centre Pompidou.

- Boulez, P. (1999). Répons, Ensemble Intercontemporain. CD Audio, DG 457 6052.

- Buxton, W. A. S. (1987). The Haptic Channel. In R. M. Baecker and W. A. S. Buxton (Eds.), Readings in Human-Computer Interaction: A Multidisciplinary Approach. San Mateo, CA: Morgan Kaufmann, 357-365.

- Camurri, A., Coletta, P., Ricchetti, M. \& Volpe, G. (2000). Synthesis of Expressive Movement. Proceedings of the 2000 International Computer Music Conference. San Francisco, CA: International Computer Music Association.

- Card, S. K., Mackinlay, J. D. \& Robertson, G. G. (1991). A Morphological Analysis of the Design Space of Input Devices. ACM Transactions on Information Systems, 9(2), 99-122.

- Cascone, K. (2000). The Aesthetics of Failure: Post-Digital Tendencies in Contemporary Computer Music. Computer Music Journal, 24(4), 12-18.

- Casserley, L. (2000). Book review of Todd Winkler, Composing Interactive Music - Techniques and Ideas Using max. The MIT Press, Cambridge, MA, Organised Sound, 5(1), 53-55.

- Cassiodorus, M.A. (1980). Institutiones. Trans. H. D. Goode \& G. C. Drake. Colorado Springs: Colorado College Music.

- Chadabe, J (1984). Interactive Composing: An Overview. Computer Music Journal, 8(1), 22-28.

- Chadabe, J. (1997). Electric Sound: The Past and Promise of Electronic Music. Upper Saddle River, New Jersey: Prentice Hall.

- Collins, N. (1991). Low Brass: Trombone-Propelled Electronics. Leonardo Music Journal, 1.

- Collins, N. (1992). It Was a Dark and Stormy Night, CD Audio, Trace Elements.

- Collins, N. (2002) Relating Superhuman Virtuosity to Human Performance. Proceedings of MAXIS, Sheffield Hallam University, Sheffield, UK, April, 2002.

- Cook, P. R. \& Leider, C. (2000). SqueezeVox: a new controller for vocal synthesis models. Proceedings of the 2000 International Computer Music Conference. San Francisco, CA: International Computer Music Association.

- Cook, P. R. (2001). Principles for Designing Computer Music Controllers. Proceedings of the 2001 Workshop on New Interfaces for Musical Expression, CHI, 2001. Seattle, WA, USA: electronic proceedings. 
- Cook, P. R., (2003). Remutualizing the Instrument: Co-design of synthesis algorithms and controllers. Proceedings of the Stockholm Music Acoustics Conference 2003 (SMAC 2003). Stockholm.

- Cutler, M., Robair, G. \& Bean (2000). OuterLimits, Electronic Musician Magazine, August 2000, 49-72.

- Dannenberg, R. \& Amon, D. (1989). A Gesture Based User Interface Prototyping System. Proceedings of the Second Annual ACM SIGGRAPH Symposium on User Interface Software and Technologies, 127-132.

- Feller, R. (2002). FMOL Trio: Live at Metronom. Computer Music Journal, 26(2), 110-112.

- Fels, S. (1994). Glove-Talk II: Mapping hand Gestures to Speech using Neural Networks - An Approach to Building Adaptive Interfaces. Ph D Thesis, University of Toronto, Canada.

- Fitts, P. M. (1951). Engineering Psychology and Equipment Design. In S. S. Stevens (Ed.), Handbook of Experimental Psychology. NY: Wiley, 1287-1340.

- Fitts, P. M. (1954). The information capacity of the human motor system in controlling the amplitude of movement. Journal of Experimental Psychology, 47, 381-391.

- Fitts, P. M., \& Posner, M. I. (1967). Human Performance. Belmont, CA: Brooks/Cole.

- Gagne, C. (1993). Interview with Laurie Spiegel. In Soundpieces 2: Interviews With American Composers. New Jersey: The Scarecrow Press, Inc., 295-333.

- Gillespie, B. 1999. Haptic Manipulation. In P. Cook (Ed.). Music Cognition and Computerized Sound: An Introduction to Psychoacoustics. Cambridge, MA: The MIT Press, 247-260

- Hansen, K.F. (2002). The basics of Scratching. Journal of New Music Research, 31(4), 357-367.

- Hornbostel, E M. \& Sachs, S. [1914] (1961). Systematik der Musikinstrumente: Ein Versuch. Zeitschrift für Ethnologie 45, 3-90, 553-90. Translated by A. Blaines and K. Wachsmann, under the title A Classification of Musical Instruments. Galpin Society Journal, 14, 3-29.

- Hunt, A. (1999). Radical User Interfaces for Real-time Musical Control. PhD Thesis, University of York UK.

- Hunt, A., Wanderley, M. M. \& Kirk, R. (2000). Towards a model for instrumental mapping in expert musical interaction. Proceedings of the International Computer Music Conference. San Francisco, CA: International Computer Music Association, 209-212.

- Jordà, S. (1991). A Real-Time MIDI Composer and Interactive Improviser by Means of Feedback Systems. Proceedings of the 1991 International Computer Music Conference, 463-466. San Francisco, CA: International Computer Music Association.

- Jordà, S. (1996). EPIZOO: Cruelty and Gratuitousness in Real Virtuality. Proceedings of 5CYBERCONF, fifth International Conference on Cyberspace [On- line]. Available: http://www.telefonica.es/fat/ejorda.html.

- Jordà, S. (2001). Improvising with Computers: A personal Survey (1989-2001). Proceedings of the 2001 International Computer Music Conference. San Francisco, CA: International Computer Music Association. 
- Jordà, S. (2002a). Improvising with Computers: A personal Survey (1989-2001). Journal of New Music Research, 31(1), 1-10.

- Jordà, S. (2002b). Afasia: the Ultimate Homeric One-man-multimedia-band. Proceedings of the 2002 Conference on New Interfaces for Musical Expression (NIME-02), 132-137. Dublin.

- Jordà, S. (2002c). FMOL: Toward User-Friendly, Sophisticated New Musical Instruments. Computer Music Journal, 26(3), 23-39.

- Jordà, S. (2003a). Sonigraphical Instruments: From FMOL to the reacTable*. Proceedings of the 2003 Conference on New Interfaces for Musical Expression (NIME-03), 70-76. Montreal.

- Jordà, S. (2003b). Interactive Music Systems For Everyone: Exploring Visual Feedback As a Way for Creating More Intuitive, Efficient and Learnable Instruments. Proceedings of Stockholm Music Acoustics Conference (SMAC03) 681-684. Stockholm.

- Kartomi, M. (1990). On Concepts and Classifications of Musical Instruments. Chicago: The University of Chicago Press.

- Kato, H., Billinghurst, M. \& Poupyrev, I. (2000). Virtual Object Manipulation on a Table-top AR Environment. Proceedings of ISAR.

- Lee, M., Freed, A. \& Wessel, A (1991). Realtime Neural Network Processing of Gestural and Acoustic Signals. Proceedings of the 1991 International Computer Music Conference, 277-280. San Francisco, CA: International Computer Music Association.

- Lehman, A. C. (1997). The Adquisition of Expertise in Music: Efficiency of Deliberate Practice as a Moderating Variable in Accounting for Sub-Expert Performance. In I. Deliège and J. A. Sloboda (Eds.), Perception and Cognition of Music. Hove, east Sussex: Psycology Press, 161-187.

- Levitin, D. J., \& Adams, R. L. (1998). Computer-Interaction Design and Controllers: Creating Meaningful Experience. Technical Report, IRC 1998-005. Palo Alto: Interval Research Corporation.

- Levitin D.J., Mcadams, S., Adams, R. L. (2002). Control parameters for musical instruments: a foundation for new mappings of gesture to sound. Organised Sound, 7(2), 171-189.

- Lewis, G. E. (2000). Too Many Notes: Computers, Complexity and Culture in Voyager. Leonardo Music Journal, 10, 33-39.

- Machover, T. (1992). Hyperinstruments - a Composer's Approach to the Evolution of Intelligent Musical Instruments. In L. Jacobson (Ed.), Cyberarts: Exploring Arts and Technology. San Francisco: MillerFreeman Inc., 67-76.

- Machover, T. (2002). Instruments, Interactivity, and Inevitability. Proceedings of the 2002 Conference on New Interfaces for Musical Expression (NIME-02), Keynote-1. Dublin.

- Macleod, M., Bowden, R. \& Bevan, N. (1997). The MUSiC Performance Measurement Method. Behaviour and Information Technology, 16.

- Marino, G., Raczinski, J.M. \& Serra, M.H. (1990). The new UPIC system Proceedings of the 1990 International Computer Music Conference. San Francisco, CA: International Computer Music Association.

- Marrin, T. A. (2000). Inside the Conductor's Jacket: Analysis, Interpretation and Musical Synthesis of Expressive Gesture. PhD Thesis, Massachussets institute of Technology. 
- Mathews, M. V. (1991). The Radio Baton and the Conductor Program, or: Pitch, the Most Important and Least Expressive Part of Music. Computer Music Journal 15(4), 37-46.

- Mathews, M. V., \& Moore, R. (1969). GROOVE, a Program for Realtime Control of a Sound Synthesizer by a Computer. In Proceedings of the 4th Annual Conference of the American Society of University Composers, NY: ASUC, Columbia University, 22-31.

- Mayr, E. (1982). The growth of biological thought: Diversity, evolution and inheritance. Cambridge, MA, and London: Belknapp Press.

- Menzies, D. (2002). Composing instrument control dynamics. Organised Sound 7(3), 255-266.

- Miller, G. A. (1956). The magical number seven plus or minus two: Some limits on our capacity for processing information. Psychological Review, 63, 81-97.

- Moore, F. R. 1988. The Disfunctions of MIDI. Computer Music Journal, 12(1), 19-28.

- Morales-Manzanares, R., Morales, E. F., Dannenberg, R. \& Berger, R. (2001). SICIB: An Interactive Music Composition System Using Body Movements. Computer Music Journal, 25(2), 25-36.

- Mulder, A. (1994). Virtual Musical Instruments: Accessing the Sound Synthesis Universe as a Performer. Proceedings of the first Brazilian Symposium on Computer Music.

- Mulder, A. (1998). Design of Gestural Constraints using Virtual Musicial Instruments. PhD thesis, School of Kinesiology, Simon Fraser University, Canada.

- Nies, J. The Art of Circuit Bending [On-line]. Available: http://www.klangbureau.de/cb_E.html

- Nishimoto, K., Oshima, C. \& Miyaga wa, Y. (2003). Why Always Versatile?: Dynamically Customizable Musical Instruments Facilitate Expressive Performances. Proceedings of the 2003 Conference on New Interfaces for Musical Expression (NIME-03), 164-169. Montreal.

- Norman, D. A. (1990). The Design of Everyday Things. NY: Doubleday.

- Nyman, M. (1980). Experimental Music: Cage and Beyond. New York: Schirmer Books.

- Orio. N. (1999). Metodi di Interfacciamento Utente-Elboratore basati sull'Analisi del Signale Audio. $\mathrm{PhD}$ thesis, Universita di Padova.

- Paine, G. (2002). Interactivity, where to from here? Organised Sound 7(3), 295304.

- Paradiso, J. \& Hsiao, K. (2000). Musical Trinkets: New Pieces to Play. SIGGRAPH 2000 Conference Abstracts and Applications. NY: ACM Press.

- Paradiso, J. (1997). Electronic Music: New ways to play. IEEE Spectrum, 34(12), 8-30.

- Patten, J., Recht, B. \& Ishii, H. (2002). Audiopad: A Tag-based Interface for Musical Performance. Proceedings of the 2002 Conference on New Interfaces for Musical Expression (NIME-02), 11-16. Dublin.

- Polansky, L., Rosenboom, D. \& Burk, P. (1987). HSML Overview (Version 3.1) and Notes on Intelligent Instrument Design. Proceedings of the 1987 International Computer Music Conference, 220-227. San Francisco, CA: International Computer Music Association. 
- Poschardt, U. (1995). Dj-Culture. London: Quartet Books Ltd.

- Poupyrev, I, (2000). Augmented Groove: Collaborative Jamming in Augmented Reality. ACM SIGGRAPH 2000 Conference Abstracts and Applications. NY: ACM Press.

- Praetorius, M. (1980). The syntagma musicum. Vol. 2, De organographica, first and second parts. Trans. H. Blumenfeld. Reprint, NY: Da capo Press.

- Pressing, J. (1990). Cybernetic Issues in Interactive Performance Systems. Computer Music Journal, 14(1), 12-25.

- Reinhard, K. (1960). Beitrag zu einer neuen Systematik der Musikinstrumente. Die Musikforschung 13, 160-64.

- Roads, C. (1986). The Second STEIM Symposium on Interactive Composition in Live Electronic Music. Computer Music Journal 10(2), 44-50.

- Roads, C. (1989). An Interview with Max Mathews. Reprinted in C. Roads, ed. The Music Machine. Cambridge, MA: The MIT Press, 5-12.

- Roads, C. (1996). Computer Music Tutorial. Cambridge, MA: The MIT Press.

- Roads, C. (2001a). Microsound. Cambridge, MA: The MIT Press.

- Roads, C. (2001b). Sound Composition with Pulsars. Journal of the Audio Engineering Society, 49(3).

- Robson, D. (2002) PLAY!: Sound Toys For Non-Musicians. Computer Music Journal, 26(3), 50-61.

- Rovan, J., Wanderley, M. M., Dubnov, S. \& Depalle, P. (1997). Instrumental Gestural Mapping Strategies as Expressivity Determinants in Computer Music Performance. In Proceedings of the Kansei -The Technology of Emotion Workshop, Genova - Italy, Oct. 1997.

- Rowe, R. (1993). Interactive Music Systems: Machine Listening and Composing. Cambridge, MA: The MIT Press.

- Rowe, R. (2001). Machine Musicianship. Cambridge, MA: The MIT Press.

- Rubine, D. \& McAvinney, P. (1990). Programmable Finger-tracking Instrument Controllers. Computer Music Journal, 14(1), 26-42.

- Rubine, D. (1991). The Automatic Recognition of Gestures. PhD thesis, Computer Science, Carnegie-Mellon University, School of Computer Science, 1991.

- Ryan, J. 1991. Some Remarks on Musical Instrument Design at STEIM. Contemporary Music Review 6(1),3-17.

- Sachs, C. (1940). The history of musical instruments. NY: Norton.

- Schloss, W. A. \& Jaffe, D. A. (1993). Intelligent Musical Instruments: The Future of Musical Performance or the Demise of the Performer? Interface Journal of New Music Research.

- Serafin, S., Dudas, R., Wanderley, M. M. \& Rodet, X. (1999). Gestural Control of a RealTime Physical Model of a Bowed String Instrument. Proceedings of the 1999 International Computer Music Conference, 375-378. San Francisco, CA: International Computer Music Association.

- Settel, Z. \& Lippe, C. (2003). Convolution Brother's Instrument Design. Proceedings of the 2003 Conference on New Interfaces for Musical Expression (NIME-03), 197-200. Montreal.

- Shannon, C. E. (1948). A mathematical theory of communication. Bell Systems Technical Journal, 27(3), 379-423, July 1948. Continued 27(4), 623-656, October 1948. 
- Shapiro, P. (1999). The Primer: Turntablism. The Wire, 179, 40-45.

- Smith, G. M., Goldstein, M. H., Eichenseer, J. W., Brook, M. B., \& Adams, R. L. (2000). System and Method for Controlling a Music Synthesizer. USA: Interval Research Corp, Patent number 6,018,118.

- Smyth, T., \& Smith, J. O. III. (2001). Applications of bioacoustics in physical modeling and the creation of new musical instruments. In Proc. of ISMA 2001, Int. Symposium on Musical Acoustics, Perugia, Italy.

- Sundberg, J. 1987. The Science of the Singing Voice. Dekalb, Illinois: Northern Illinois University Press.

- Timoshenko, S. \& Young, D. (1937). Engineering Mechanics - Statics. McGraw-Hill.

- Tone, Y. (1997) Solo for Wounded CD. CD Audio, Tzadik \#7212.

- Ulyate, R. \& Bianciardi, D. (2002). The Interactive Dance Club: Avoiding Chaos in a Multi-Participant Environment. Computer Music Journal, 26(3), 4049.

- Ungvary, T., \& Vertegaal, R. (1999). The SensOrg: time-complexity and the design of a musical cyberinstrument. Proceedings of the 1999 International Computer Music Conference, 363-366. San Francisco, CA: International Computer Music Association.

- Vertegaal, R. \& Eaglestone, B. (1996). Comparison of input devices in an ISEE direct timbre manipulation task. Interacting with Computers, 8(1), 13-30.

- Vertegaal, R., Ungvary, T., \& Kieslinger, M (1996). Towards a musician's cockpit: transducers, feedback and musical function. Proceedings of the 1996 International Computer Music Conference, 308-311. San Francisco, CA: International Computer Music Association.

- Waisvisz, M. (1985). The Hands, a set of Remote MIDI-controllers. In Proceedings of the 1985 International Computer Music Conference, 313-318. San Francisco, CA: International Computer Music Association.

- Waisvisz, M. (1999). On Gestural Controllers. http://www.crackle.org/ MW's\%20gestural\%20round\%20table.htm

- Wanderley, M. M. \& M. Battier, eds. (2000). Trends in Gestural Control of Music (CD-ROM). Paris: Ircam-Centre Pompidou.

- Wanderley, M. M. \& Orio, N. (2002). Evaluation of Input Devices for Musical Expression: Borrowing Tools from HCI. Computer Music Journal, 26(3), 62-76.

- Wanderley, M. M. (2001). Performer-Instrument Interaction: Applications to Gestural Control of Music. PhD Thesis. Paris: University Pierre et Marie Curie Paris VI.

- Wang, G. \& Cook, P.R. (2004). On-the-fly Programming: Using Code as an Expressive Musical Instrument. Proceedings of the 2004 Conference on New Interfaces for Musical Expression (NIME-04). Hamamatsu, Japan.

- Weaver, W. \& Shannon C. E. (1949), The Mathematical Theory of Communication. Urbana, Illinois: University of Illinois Press, 1949, republished in paperback 1963.

- Weinberg, G., \& Gan, S. (2001). The Squeezables: Toward an Expressive an Interdependent Multi-Player Musical Instrument. Computer Music Journal, 25(2), 37-45.

- Weinberg, G., \& Jennings, K. (2002). The Beatbug Network - A rythmic System for Interdependent Group Collaboration. Proceedings of the 2002 
Conference on New Interfaces for Musical Expression (NIME-02), 107-111. Dublin.

- Wessel, D. \& Wright, M. (2002). Problems and Prospects for Intimate Musical Control of Computers Computer Music Journal, 26(3), 11-22.

- Winkler, T. (1995). Making Motion Musical: Gesture Mapping Strategies for Interactive Computer Music. Proceedings of the 1995 International Computer Music Conference. San Francisco, CA: International Computer Music Association.

- Winkler, T. (1998). Composing Interactive Music: Techniques and Ideas Using Max. Cambridge, MA: The MIT Press.

- Winnington-Ingram, R. P. (1980). Greece, ancient. In The new Groove dictionary of music and musicians, ed. S. Sadie, 7, 659-72. London: Macmillan.

- Xenakis, I (1992). Formalized Music. NY: Pendragon Press. 